\title{
CARACTERIZAÇÃO DO PRÉ-DESENVOLVIMENTO DO PSS: ESTUDO DE CASOS EM EMPRESAS FABRICANTES DE EQUIPAMENTOS MÉDICO- HOSPITALARES
}

Francisco Andrea Simões Braga (franciscoasb@uniara.com.br) - Universidade de Araraquara (UNIARA)

Glauco Mendes (glauco@dep.ufscar.br) - Departamento de Engenharia de Produção / Universidade Federal de São Carlos

\section{RESUMO}

O Sistema Produto-Serviço (PSS) corresponde à oferta integrada de produtos e serviços e consiste numa abordagem que permite inovar a forma de gerar valor ao cliente. A oferta de PSS têm sido uma realidade para fabricantes de equipamentos médico-hospitalares, que têm sido compelidos a desenvolver produtos, serviços e soluções que favoreçam o aumento da qualidade e a redução dos custos na área de saúde. Há diversas metodologias para o desenvolvimento de PSS, porém o desafio é compreender as especificidades do desenvolvimento PSS, especialmente os ligados à fase de pré-desenvolvimento. Este trabalho tem objetivo de descrever o pré-desenvolvimento do PSS em empresas fabricantes de equipamentos médicohospitalares. Foi realizado uma pesquisa bibliográfica e estudo de casos em duas empresas do setor. Os principais resultados evidenciaram que empresas não possuem um prédesenvolvimento estruturado. Além disso há pouca ênfase no desenvolvimento dos serviços ofertados pela empresa. O artigo contribui em i) apresentar um tipo de especial de PSS em empresas do setor de equipamento médico-hospitalares (comodato); ii) apontar que, diferentemente, da literatura, as empresas investigadas possuem processos não-estruturados com pouca ênfase no desenvolvimento dos serviços.

Palavras chave: sistema produto-serviço, pré desenvolvimento, equipamentos médicohospitalares. 


\section{INTRODUÇÃ̃}

Observa-se um aumento significativo nos gastos com saúde no Brasil, que, atualmente, é de cerca de 8\% do PIB (OPAS/OMS, 2017). Diante desse cenário, muitos países estão engajados na redução desses gastos. Uma das estratégias é substituição de compras de equipamentos por compras de serviços, o que tem levado os fabricantes de equipamentos médicos a investir não só em novas tecnologias médicas, mas também em serviços de saúde mais eficientes (YIP; PHAAL; PROBERT, 2014).

Dentre as alternativas que podem ser adotadas pelos fabricantes de equipamentos médico está o Sistema Produto-Serviço (no artigo, usaremos a expressão Product-Service System - PSS), que é definido como uma oferta integrada de produtos e serviços que entrega valor em uso. $\mathrm{O}$ PSS oferece uma oportunidade para separar sucesso econômico do consumo material, o que, consequentemente, pode também reduzir o impacto ambiental das atividades econômicas (BAINES et al., 2007). Todavia, a mudança da oferta de produtos para a oferta do PSS requer mudanças relacionada à forma como o PSS é desenvolvido (TAN, 2010).

O processo de desenvolvimento de PSS contempla uma fase inicial crítica, que é a macrofase de pré-desenvolvimento. Tendo origem nos processos de desenvolvimento de produto, o Fuzzy Front-End (FFE) (SMITH; REINERTSEN, 1998), posteriormente chamado de Front-End of Innovation (FEI) é uma fase de planejamento composta por atividades realizadas antes do desenvolvimento de tecnologias, produtos e/ou serviços ou novos negócios de uma organização (KOEN, 2001). Fundamental para o processo de FEI são as atividades de geração e triagem de ideias, geração de conceito, análises de viabilidades e planejamento do projeto. Também informações disponíveis, muitas vezes incertas ou insuficientes (OLIVEIRA et al., 2015; KHURANA; ROSENTHAL, 1998; KOEN et al., 2001; MOENAERT et al., 1995) Além das informações no processo de decisão, outro fator fundamental para esta análise são os critérios que norteiam o processo de decisão (CARBONELL-FOULQUIÉ et al., 2004; COOPER et al., 2001; MOENAERT et al., 2010).

No caso do PSS, o pré-desenvolvimento do PSS é um desafio para as empresas manufatureiras, ainda mais quando os tipos de PSS orientados ao uso e orientados aos resultados são os adotados. Neste caso, sugere-se que tanto o desenvolvimento do produto como o desenvolvimento do serviço devem ser integrados. Ademais, a oferta desses tipos de PSS pode representar a criação de um novo modelo de negócio (TAN, 2010). 
A partir deste contexto, observa-se a oportunidade de pesquisar a respeito da fase inicial do desenvolvimento de PSS. Logo, o objetivo deste trabalho é descrever o pré-desenvolvimento do desenvolvimento do PSS em duas empresas fabricantes de equipamentos médicohospitalares.

Como método de pesquisa, foram realizados estudos de casos em duas empresas fabricantes de equipamentos médico-hospitalares. Os dados foram coletados por meio de entrevistas semiestruturadas com representantes das áreas de engenharia e comercial das empresas investigadas. $\mathrm{O}$ artigo contribui em i) apresentar um tipo de especial de PSS em empresas do setor de equipamento médico-hospitalares (comodato); ii) apontar que, diferentemente, da literatura, as empresas investigadas possuem processos não-estruturados com pouca ênfase no desenvolvimento dos serviços.

\section{REVISÃO TEÓRICA}

\subsection{Sistema Produto-Serviço (PSS)}

Na oferta de PSS, empresas de manufatura adotam uma postura mais orientada aos serviços e, portanto, investem em serviços com o objetivo de satisfazer as necessidades do cliente, obter vantagens competitivas e melhorar seu próprio desempenho. A oferta de PSS está relacionada à tendência de servitização (KOWALKOWSKI; GEBAUER; OLIVA, 2017). Para as empresas que adotam a servitização, é exigido novas estratégias de serviços (GEBAUER et al., 2010), novas capacidades (ELORANTA; TURUNEN, 2015) e novas estruturas e recursos organizacionais (GEBAUER; FISCHER; FLEISCH, 2010). A partir da servitização, há a concepção de um novo modelo de negócio, cuja oferta consiste num pacote integrado de produtos e serviços chamado Sistema Produto-Serviço (PSS) (BAINES et al., 2007).

Há três tipos clássicos de tipos de PSS que podem ser ofertados ao cliente, conforme descreve Tukker (2004):

- PSS orientado a produto: há a venda do produto de forma tradicional, incluindo o ato de venda de serviços adicionais, tais como o serviço pós-venda (exemplo: manutenção, reparação, reutilização e reciclagem) para garantir funcionalidade e durabilidade do produto detido pelo cliente. 
- PSS orientado ao uso: há a venda do uso ou a disponibilidade de um produto, portanto, não há transferência da propriedade do cliente (por exemplo, leasing, compartilhamento). Neste caso, a empresa é motivada a criar um PSS para maximizar o uso do produto necessário para atender à demanda e estender a vida útil do produto e materiais utilizados para produzi-lo.

- PSS orientado ao resultado: há a venda de um resultado ou capacidade ao cliente em vez de um produto (por exemplo, vendendo roupas lavadas em vez de uma máquina de lavar). As empresas oferecem uma combinação personalizada de serviços onde o produtor mantém a propriedade do produto e o cliente paga apenas pela provisão de resultados acordados.

\subsection{Modelos de Desenvolvimento de PSS}

Na tentativa de auxiliar empresas desenvolverem PSS, muitos autores propuseram modelos para o processo de desenvolvimento de PSS (MDPSS) (MARILUNGO; PERUZZINI; GERMANI, 2016). Muitos desses modelos foram elaborados com base em metodologias tradicionais das áreas de desenvolvimento de produto ou de serviços, admitindo-se que a simples expansão desses modelos é suficiente para permitir integração de produtos e serviços. Neste artigo, alguns dos principais MDPSSs são apresentados.

O primeiro é o Service Model, sendo considerado um modelo para o desenvolvimento integrado de produtos e serviços a fim de contribuir para a produção e consumo sustentáveis (SAKAO; SHIMOMURA, 2007). Outro modelo clássico é o Methodology for Product-service System (MEPSS), que busca guiar as empresas no desenvolvimento e implantação de sistemas produtoserviços também com foco na sustentabilidade. O MEPSS adota uma abordagem modular, permitindo que a empresa inicie suas atividades de desenvolvimento em diferentes fases do modelo, dependendo do atual estágio do seu projeto de PSS serviços (VAN HALEN; VEZZOLI; WIMMER, 2005). Outro modelo é o Total Care Products (TCP), que divide o processo iterativo cliente-fornecedor em cinco estágios: i) Estágio 1 (ambições de negócio dos clientes); ii) Estágio 2 (definição de soluções potenciais); iii) Estágio 3 (identificação da solução principal); iv) Estágio 4 (detalhamento da solução principal); v) Estágio 5 (análise de risco e validação do negócio) (ALONSO-RASGADO; THOMPSON, 2006). Além desses, existe o Integrated Product and Service Design Processes (IPSDP) que permite, a partir do processo de desenvolvimento de produto, agregar as atividades de desenvolvimento dos serviços, gerando a criação de um processo integrado de desenvolvimento de produtos e serviços (AURICH; FUCHS; DEVRIES, 2004). 
Os MDPSS também podem ser divididos em pré-desenvolvimento, desenvolvimento e pósdesenvolvimento. Porém, alguns modelos possuem a fase de pré-desenvolvimento mais evidente, como é o caso do MEPSS, sendo este composto por cinco principais fases: análise estratégica, exploração de oportunidades, desenvolvimento da ideia do PSS, desenvolvimento do conceito do PSS e desenvolvimento e implantação do projeto de PSS. Entre as fases, existem os pontos de decisão que funcionam como momentos de avaliação e decisão, nos quais são verificados os resultados alcançados pelo projeto até aquele momento, o alinhamento com as estratégias e recursos da empresa e a viabilidade de condução do projeto dos pontos de vista técnico e econômico. Com base nessas avaliações, a equipe responsável pelo projeto de PSS decide prosseguir, ou não, com o esforço de desenvolvimento (VAN HALEN; VEZZOLI; WIMMER, 2005). No caso do Integrated Product and Service Design Processes (IPSDP) (AURICH; FUCHS; DEVRIES, 2004), o pré-desenvolvimento engloba as atividades tradicionais propostas por Cooper (1988), tais como : i) o Estágio I (Ideia) envolve atividades de geração e triagem de ideia; ii) o Estágio II (Avaliação Preliminar) engloba atividades de avaliação preliminar de mercado e técnica e iii) o Estágio III (Definição do Conceito) aborda as atividades de identificação, definição, teste e avaliação do conceito.

\section{MÉTODO DE PESQUISA}

O método de pesquisa adotado neste trabalho é o estudo de caso, pois mostra-se como sendo o mais adequado para responder questões "como" e "por que"; situações onde o investigador tem pouco controle sobre os eventos; o enfoque está em um fenômeno contemporâneo no contexto da vida real, conforme descreve Yin (2015). Para tanto, foram estudadas duas empresas (Empresa A e B) fabricantes de equipamentos médico-hospitalares, que além de fabricar produtos, também oferecem serviços associados a eles.

A Empresa A é focada em sistemas de infusão hospitalar com mais de 56 modelos distintos de aplicação e é considerada a líder do seu segmento. Possui duas unidades de negócio situadas no Estado de São Paulo e conta com aproximadamente 300 colaboradores diretos. Os principais clientes da empresa são hospitais de médio a grande porte, públicos ou privados. O produto objeto de estudo é um equipamento conhecido como bomba de infusão. Já a Empresa B tem como foco a fabricação de equipamentos para estudo Urodinâmico. Seus produtos são fabricados no Brasil e comercializados em todo território nacional. Está situada na cidade de 
São Paulo e conta com aproximadamente 30 colaboradores. Os principais clientes da empresa são hospitais de médio a grande porte privados. O produto objeto de estudo é um equipamento de diagnóstico para urologia.

A coleta de dados se deu por meio da realização de entrevistas semiestruturadas baseadas em um roteiro com perguntas principais com questões abertas e fechadas. Para construção do roteiro de entrevistas, dois construtos foram relacionados: caracterização do PSS e prédesenvolvimento do processo de PSS. O roteiro de entrevista foi composto por quatro blocos, cobrindo os seguintes aspectos: caracterização da empresa e do(s) entrevistado(s); caracterização do PSS estudado; pré-desenvolvimento do PSS. Além das entrevistas, outras fontes foram consultadas, tais como documentos e website das empresas.

A análise dos dados aconteceu em quatro etapas: 1) transcrição literal das entrevistas para arquivos digitais, onde foram coletados $4,0 \mathrm{~h}$ de áudios a partir de entrevistas com 3 colaboradores das duas empresas; 2) agrupamento de informações coletadas por meio de observações, onde as entrevistas e anotações realizadas durantes as entrevistas foram inseridos e organizados em um software de análise qualitativa, no caso o Atlas.ti; 3 ) redução manual dos dados por meio do processo de análise de conteúdo para cada categoria proveniente dos constructos definidos; 4) cruzamento das evidências coletadas entre os casos.

\section{RESULTADOS E DISCUSSÃO}

\section{1 - Caracterização do PSS das empresas estudadas}

Nas duas empresas, o modelo de negócio adotado é o comodato. Trata-se de um PSS orientado ao uso, no qual o equipamento é disponibilizado gratuitamente ao cliente. Porém, há um contrato de fornecimento de insumos, em que se estipula uma quantidade mínima de insumos a ser consumida, dando direito ao uso do equipamento. No comodato, a propriedade do equipamento é da empresa. A motivação da adoção do PSS pela Empresa A se deu pelo alto retorno financeiro pela venda dos insumos. Já para a Empresa B, o PSS foi motivado por permitir a disponibilização de seu produto em hospitais de referência, sendo uma espécie de publicidade gratuita.

Dentre os serviços ofertados, estão a instalação, manutenção, treinamento de uso e calibração do equipamento. Quanto aos tipos de PSS e serviços associados, observa-se que nas duas empresas, os serviços associados são semelhantes, compreendendo, basicamente, a manutenção 
e o treinamento de operação dos equipamentos. Também, não foi observada a oferta de serviços avançados, tais como operação ou garantia de desempenho do produto. O Quadro 1 apresenta uma síntese das características gerais do PSS das Empresas A e Empresa B.

Quadro 1 - Síntese dos resultados - Caracterização do PSS

\begin{tabular}{|c|c|c|}
\hline Categorias & Empresa A & Empresa B \\
\hline Tipo de PSS & Comodato (Orientado ao uso) & Comodato (Orientado ao uso) \\
\hline $\begin{array}{l}\text { Tipo de Serviços associados } \\
\text { Instalação }\end{array}$ & & $\checkmark$ \\
\hline Manutenção & $\checkmark$ & $\checkmark$ \\
\hline \multicolumn{3}{|l|}{ Operação } \\
\hline \multicolumn{3}{|l|}{ Diagnóstico } \\
\hline \multicolumn{3}{|l|}{ Consultoria } \\
\hline Treinamento & $\checkmark$ & $\checkmark$ \\
\hline Calibração & $\checkmark$ & \\
\hline \multicolumn{3}{|l|}{ Propriedade do Produto } \\
\hline Produtor / Provedor & $\checkmark$ & $\checkmark$ \\
\hline $\begin{array}{l}\text { Motivação para adoção do } \\
\text { PSS }\end{array}$ & Alto lucro & $\begin{array}{l}\text { Ter o produto em hospitais de } \\
\text { referência }\end{array}$ \\
\hline $\begin{array}{l}\text { Vantagens da adoção do } \\
\text { PSS }\end{array}$ & Retorno financeiro & Publicidade \\
\hline $\begin{array}{l}\text { Desvantagens da adoção } \\
\text { do PSS }\end{array}$ & $\begin{array}{c}\text { - Aumento dos ativos } \\
\text { - Baixa qualidade do produto } \\
\text { fabricado }\end{array}$ & $\begin{array}{c}\text { - Baixa venda dos insumos utilizados } \\
\text { no produto }\end{array}$ \\
\hline Tempo de adoção do PSS & 30 anos & 4 anos \\
\hline
\end{tabular}

\section{2 - Pré-desenvolvimento do PSS nas empresas estudadas}

Esta seção descreve como a Empresa A e a Empresa B executam suas atividades de prédesenvolvimento. O nível de maturidade do pré-desenvolvimento nas duas empresas é baixo, caracterizado pela informalidade e pela baixa utilização de métodos estruturados de planejamento e tomada de decisão. Principalmente, há pouca (quase nenhuma) ênfase no desenvolvimento e integração dos serviços associados aos equipamentos cedidos. Assim, a integração entre desenvolvimento de produto e desenvolvimento de serviços representa um grande desafio para as empresas que buscam implantar modelos de negócios pautados no PSS. O Quadro 2 apresenta uma síntese das características gerais do pré-desenvolvimento das Empresas A e Empresa B. 
Quadro 2 - Síntese dos resultados - Caracterização do PSS

\begin{tabular}{|c|c|c|}
\hline Categorias & Empresa A & Empresa B \\
\hline $\begin{array}{l}\text { Desenvolvimento integrado } \\
\text { de produto e serviço }\end{array}$ & Não & Não \\
\hline $\begin{array}{l}\text { Desenvolvimento } \\
\text { produto }\end{array}$ & Não-estruturado e informal & Não-estruturado e informal \\
\hline Modelo de Referência & $\begin{array}{c}\text { ABNT NBR ISO } 13485 \text { e RDC } \\
16 \text { da ANVISA }\end{array}$ & $\begin{array}{c}\text { ABNT NBR ISO } 13485 \text { e RDC } 16 \\
\text { da ANVISA }\end{array}$ \\
\hline $\begin{array}{l}\text { Desenvolvimento } \\
\text { serviço }\end{array}$ & Não existente & Não existente \\
\hline Responsabilidade & Engenharia (produto) & Engenharia (produto) \\
\hline desenvolvimento & Comercial (serviços) & Comercial (serviços) \\
\hline \multicolumn{3}{|l|}{ Pré-desenvolvimento } \\
\hline $\begin{array}{l}\text { Avaliação preliminar de } \\
\text { mercado }\end{array}$ & $\begin{array}{l}\text { Engenharia (requisitos do } \\
\text { produto) }\end{array}$ & $\begin{array}{l}\text { Comercial (informações mercado, } \\
\text { tendências, pesquisas clientes) }\end{array}$ \\
\hline Geração da Ideia & $\begin{array}{c}\text { De forma espontânea } \\
\text { Diversas fontes (clientes, } \\
\text { produtos concorrentes) }\end{array}$ & $\begin{array}{l}\text { De forma informal } \\
\text { Engenharia }\end{array}$ \\
\hline Triagem das Ideias & $\begin{array}{l}\text { Necessidade de clientes, } \\
\text { requisitos estatutários e índice de } \\
\text { defeitos dos produtos atuais } \\
\text { Direção (aprovação) }\end{array}$ & $\begin{array}{l}\text { De forma informal } \\
\text { Direção }\end{array}$ \\
\hline Conceito Preliminar & $\begin{array}{l}\text { Engenharia (Definição dos dados } \\
\text { de entrada do projeto) }\end{array}$ & $\begin{array}{l}\text { Não registrado, mas é conhecido } \\
\text { pela Direção }\end{array}$ \\
\hline Análise de Viabilidade & $\begin{array}{c}\text { De forma informal } \\
\text { Direção (aprovação final) }\end{array}$ & $\begin{array}{l}\text { Engenharia (preço do produto, } \\
\text { disponibilidade de fornecedores) }\end{array}$ \\
\hline $\begin{array}{l}\text { Planejamento preliminar do } \\
\text { projeto }\end{array}$ & $\begin{array}{l}\text { Engenharia (Define o Plano de } \\
\text { Projeto) }\end{array}$ & $\begin{array}{l}\text { Engenharia (Planejamento e } \\
\text { controle do Projeto) }\end{array}$ \\
\hline Pontos de decisão (gates) & Sim & Sim \\
\hline
\end{tabular}

Fonte: Elaborado pelo autor

Como evidenciado nas duas empresas, não há um processo para o DPSS em nenhuma das empresas, sendo que ambas priorizam o processo de desenvolvimento de produto. Por serem empresas fabricantes de equipamentos médicos possuem certificação de seus Sistemas de Gestão da Qualidade. Logo, os requisitos de projeto e desenvolvimento são requeridos pelas normas de certificação, tais como a ABNT NBR ISO 13485 e RDC 16 da ANVISA.

Nas duas empresas, o processo de desenvolvimento de produtos é de responsabilidade do departamento de Engenharia. Ainda que existam áreas dedicadas à assistência técnica, elas se encarregam da execução dos serviços (função mais técnica e operacional). De fato, tanto na Empresa A como na Empresa B, não existe uma área dedicada e exclusiva para $\mathrm{o}$ desenvolvimento de novos serviços, sendo que a área Comercial, por ser mais próxima aos clientes assume um papel importante na geração da inovação de novos serviços. Este relacionamento é interessante para o processo por intensificar a geração de insights, facilitar a 
empresa a traduzir as demandas do mercado e também de aferir a satisfação dos clientes com os serviços e produtos disponibilizados.

Como em qualquer processo de desenvolvimento, há atividades ligadas ao pré-desenvolvimento ainda que este termo não era de conhecimento dos entrevistados. Não há estratégias claras de inovação. Apesar das duas empresas possuírem um planejamento estratégico formalizado, um plano estratégico de produto/serviço não é concebido pelas empresas. Neste sentido, a Empresa B mencionou exemplos de novos projetos que foram desenvolvidos a partir de contingências do mercado, sem que estivessem contemplados em seu planejamento. Fica evidenciada a falta de articulação formal entre as estratégias de negócio e as de produtos/serviços, prevalecendo uma estratégia emergente baseadas nas tecnologias e mercados já explorados pela empresa Quanto ao pré-desenvolvimento, as duas empresas se concentram nas atividades de identificação de oportunidades, geração de ideias e análise de viabilidade (Quadro 2). Esta ênfase na fase inicial é influenciada basicamente pelo nível de contato que as empresas estabelecem com seus clientes. A identificação de oportunidades e avaliação preliminar de mercado é, geralmente, de responsabilidade da própria diretoria ou das áreas comerciais, que vislumbram oportunidades por meio da participação em eventos do setor, análise da concorrência e em informações colhidas de clientes ou consultores por meio de contatos/relacionamentos ou pesquisas de mercado (por exemplo, Empresa B). Trata-se de um processo realizado de forma não sistemática e muito fundamentado em percepções particulares dos indivíduos das áreas próximas ao cliente. Apesar de parecer um elemento óbvio, o monitoramento contínuo dos aspectos do mercado necessitaria ser aprimorado por essas empresas.

As ideias para novos produtos/serviços são originadas a partir de diversas fontes, mas, principalmente, áreas funcionais, clientes, concorrentes, fornecedores, feiras etc. Não há programas sistematizados de geração de ideias nas Empresas A e B. Além disso, nenhum dos entrevistados alegou possuir procedimentos estabelecidos para a avaliação e seleção das ideias, sendo que as decisões são tomadas pelas respectivas Diretorias. A avaliação técnica compete às áreas de Engenharia e a avaliação econômica é feita pela área Comercial. Observou-se, nesta etapa, grande interação entre as áreas envolvidas, o que contribui para a qualidade de execução. As avaliações, porém, são frágeis e não seguem métodos mais estruturados de análise. A viabilidade técnica é feita de maneira superficial, baseada na existência ou não de recursos 
materiais e na capacidade de realização do produto. A análise financeira é realizada pelos de indicadores tradicionais (projeção de vendas, custos e lucratividade) e pautada na experiência e percepção dos executores. Existe uma preocupação maior em manter um certo nível de custo (exemplo, custos dos novos componentes incorporados aos produtos).

A aprovação final passa, predominantemente, por uma decisão tomada pela diretoria de cada uma das empresas. Na sequência, são determinadas as tarefas, as responsabilidades e os prazos para cada etapa de desenvolvimento. Foram identificados graus de detalhamento diferenciados na elaboração do projeto do produto, sendo o cronograma de atividades um de seus principais elementos. Por fim, quanto à utilização de gates, as duas empresas afirmaram utilizar mecanismos semelhantes de avaliação, mas não revelaram adotar procedimentos mais detalhados na realização das análises. De fato, constam análises críticas (pontos de verificação e validação), os quais são também requeridos pelas normas de certificação (ABNT NBR ISO 13485 e RDC 16 da ANVISA), mas que ocorrem informalmente e executadas pelos envolvidos no projeto.

\section{CONCLUSÃO}

Esse trabalho teve o objetivo de descrever o pré-desenvolvimento (Front-end of innovation) em duas empresas fabricantes de equipamentos médico-hospitalares. Para a realização da pesquisa, usou-se estudo de caso como estratégia de pesquisa a fim de esclarecer a realidade dessas empresas quanto ao uso do PSS e práticas ligadas ao seu desenvolvimento.

Em síntese, pode-se concluir que o baixo nível de maturidade do PDPSS. Nas empresas, o desenvolvimento de produto é conduzido de maneira informal e não-sistematizada. A maneira empírica baseada na experiência adquirida ao longo dos anos pelos envolvidos reforça esta postura. Além disso, observa-se uma deficiência graves com relação às práticas de desenvolvimento de novos serviços, cujo processo é praticamente inexistente nas duas empresas. Outro ponto a se destacar é que, consequentemente, o levantamento de informações para o desenvolvimento do PSS se concentra no desenvolvimento do produto. Praticamente, nada foi mencionado em relação às informações necessárias à prestação dos serviços associados aos produtos. Esses resultados mostram-se distantes do preconizados pela literatura sobre o processo de desenvolvimento do PSS. Por exemplo, sugere-se nos tipos de PSS orientados ao uso e ao resultado, que tanto o desenvolvimento do produto (DP) quanto o desenvolvimento de 
serviços (DS) devem ser integrados num mesmo processo a fim de evitar problemas de compatibilidade e dificuldades de maximização do potencial do PSS para os clientes (TAN, 2010). Além disso, a utilização de métodos e ferramentas ligadas ao desenvolvimento não é satisfatoriamente aplicada nas atividades de pré-desenvolvimento. Por fim, apesar das duas empresas terem o comodato como seu principal negócio, ambas continuam empresas focadas em produtos (product-centric). Para as empresas, os serviços não possuem centralidade nos negócios e são tratados de forma não-prioritária.

As constatações acima podem ser resultantes de limitações do trabalho. Devido ao nível de maturidade do processo de desenvolvimento do PSS das empresas estudadas, que se mostrou em um nível não elevado, o aprofundamento do conhecimento de práticas ligadas aos prédesenvolvimento do PSS foi prejudicado. A pesquisa teve como outra limitação, o baixo número de respondentes, além de não ser possível entrevistar os diretores, representantes da área comercial ou CEOs das empresas. Como forma de superar essas limitações, identifica-se como fonte de trabalhos futuros, a realização de estudos mais aprofundados com um número maior de empresas e de ramos diferentes, já que são cenários díspares e que podem corroborar ou até mesmo refutar os achados aqui apresentados.

\section{REFERÊNCIAS}

ALONSO-RASGADO, T.; THOMPSON, G. A rapid design process for Total Care Product creation. JOURNAL OF ENGINEERING DESIGN, v. 17, n. 6, p. 509-531, 2006.

AURICH, J. C.; FUCHS, C.; DEVRIES, M. F. An Approach to Life Cycle Oriented Technical Service Design. CIRP Annals - Manufacturing Technology, v. 53, n. 1, p. 151-154, 2004.

BAINES, T. S. et al. State-of-the-art in product-service systems. Proceedings of the Institution of Mechanical Engineers Part B-Journal of Engineering Manufacture, v. 221, n. 10, p. 1543-1552, 2007.

CARBONELL-FOULQUIÉ, P. et al. Criteria employed for go/no-go decisions when developing successful highly innovative products. Industrial Marketing Management, v. 33, n. 4, p. 307-316, 1 maio 2004.

COOPER, R. et al. Portfolio management for new product development: results of an industry practices study. R and D Management, v. 31, n. 4, p. 361-380, 1 out. 2001.

COOPER, R. G. Predevelopment activities determine new product success. Industrial Marketing Management, v. 17, n. 3, p. 237-247, 1988.

ELORANTA, V.; TURUNEN, T. Seeking competitive advantage with service infusion: A systematic literature review. Journal of Service Management, v. 26, n. 3, p. 394-425, 15 jun. 2015.

GEBAUER, H.; FISCHER, T.; FLEISCH, E. Exploring the interrelationship among patterns of service strategy changes and organizational design elements. Journal of Service Management, v. 21, n. 1, p. 103-129, 2010. 
KOEN, P. et al. Providing clarity and a common language to the "fuzzy front end". Research Technology Management, v. 44, n. 2, p. 46-55, 2001.

KOWALKOWSKI, C. et al. Servitization and deservitization: Overview, concepts, and definitions. Industrial Marketing Management, v. 60, p. 4-10, 1 jan. 2017.

MARILUNGO, E.; PERUZZINI, M.; GERMANI, M. Review of product-service system design methods. IFIP Advances in Information and Communication Technology. Anais...Springer, Cham, 2016Disponível em: 〈http://link.springer.com/10.1007/978-3-319-33111-9_25>. Acesso em: 18 maio. 2017

MOENAERT, R. K. et al. Strategic innovation decisions: What you foresee is not what you get. Journal of Product Innovation Management, v. 27, n. 6, p. 840-855, 1 nov. 2010.

OLIVEIRA, M. G. et al. Decision making at the front end of innovation: the hidden influence of knowledge and decision criteria. R\&D Management, v. 45, n. 2, p. 161-180, 1 mar. 2015.

OPAS/OMS. OPAS/OMS Brasil. Disponível <https://www.paho.org/bra/index.php?option=com_content\&view=article\&id=5874:paises-estaogastando-mais-em-saude-mas-pessoas-ainda-pagam-muitos-servicos-com-dinheiro-do-propriobolso\&Itemid=843>. Acesso em: 5 mar. 2019.

SAKAO, T.; SHIMOMURA, Y. Service Engineering: a novel engineering discipline for producers to increase value combining service and product. Journal of Cleaner Production, v. 15, n. 6, p. 590-604, 2007.

SMITH, P. G. et al. Developing products in half the time: new rules, new tools. John Wiley Sons, n. 2, p. 1-298, 1998.

TAN, A. R. Service-oriented product development strategies PhD thesis. [s.l.] DTU Management Engineering, 2010.

TUKKER, A. Eight Types of Product Service Systems: Eight Ways To Sustainability? Business Strategy and the Environment, v. 260, n. 13, p. 246-260, 2004.

VAN HALEN, C.; VEZZOLI, C.; WIMMER, R. Methodology for product service system innovation: How to develop clean clever and competitive strategies in companies. Royal Van Gorcum, Assen, Netherlands., 2005.

YIP, M. H.; PHAAL, R.; PROBERT, D. R. Stakeholder Engagement in Early Stage Product-Service System Development for Healthcare Informatics. Engineering Management Journal, v. 26, n. 3, p. 52-62, 2014. 